Original Research Paper

\title{
Solution of Second Order Ordinary Differential Equation Associated with Toeplitz and Stiffness Matrices
}

\author{
${ }^{1,2}$ Sami H. Altoum \\ ${ }^{l}$ AL-Qunfudhah University College, Umm Al-Qura University, KSA \\ ${ }^{2}$ Department of Mathematics, Academy of Engineering Sciences, Sudan
}

Article history

Received: 26-06-2018

Revised: 19-09-2018

Accepted: 29-10-2018

Email: samialtoum@hotmail.com

\begin{abstract}
In this work we develop a technique solution of second order ordinary differential equation (integrating by parts) to reach to Toeplitz matrices and Stiffness matrix to solve O.D.Es. We investigate that solution numerically using finite difference method and we find the error between exact and numerical solution computed using finite element.
\end{abstract}

Keywords: Differential Equation, Toeplitz Matrix, Trapezoidal Method, Stiffness Matrix

\section{Introduction}

Ordinary differential equations frequently occur as mathematical models in many branches of science, physics, chemistry, biology, engineering and economy. In the finite element method for the numerical solution of elliptic partial differential equations, the stiffness matrix represents the system of linear equations. That must be solved in order to ascertain an approximate solution to the differential equation (Chniti et al., 2016; Gavin, 2014; Ye and Lim, 2015). The stiffness matrix for other PDE follows essentially the same procedure, but it can be complicated by the choice of boundary conditions. In order to implement the finite element method on a computer, one must first choose a set of basis functions and then compute the integrals defining the stiffness matrix. Usually, the domain $\Omega$ is discredited by some form of mesh generation, where it is divided into non-overlapping triangles or quadrilaterals, which are generally referred to as elements. The basis functions are then chosen to be polynomials of some order with in each element and continuous across element boundaries. The simplest choices are piecewise linear for triangular elements and piecewise bilinear for rectangular elements. In linear algebra, a Toeplitz matrix or diagonal-const ant matrix, named after Otto Toeplitz, is a matrix in which each descending diagonal from left to right is constant. A ma- trix equation of the form $A x=$ $b$ is called a Toeplitz system if $A$ is a Toeplitz matrix. If $A$ is an $n \times n$ Toeplitz matrix, then the system has only $2 n-1$ degrees of freedom, rather than $n^{2}$. We might therefore expect that the solution of a Toeplitz system would be easier and indeed that is the case. Toeplitz systems can be solved by the Levinson algorithm in $\Theta\left(n^{2}\right)$ time. Variants of this algorithm have been shown to be weakly stable (i.e., they exhibit numerical stability for well-conditioned linear systems). The algorithm can also be used to find the determinant of a Toeplitz matrix in $O\left(n^{2}\right)$ (mathematical notation that describes the limiting behaviour of a function) time (Saoudi et al., 2018; Agarwal and El-Sayed, 2018; Zhou and Agarwal, 2017; Ruzhansky et al., 2017). A Toeplitz matrix can also be decomposed (i.e., factored) in $O\left(n^{2}\right)$ time. The Bareiss algorithm for an LU decomposition is stable. An LU decomposition (where 'LU' stands for 'lower upper' and also called LU factorization) gives a quick method for solving a Toeplitz system and also for computing the determinant. Algorithms that are asymptotically faster than those of Bareiss and Levinson ha ve been described in the literature, but their accuracy cannot be relied upon (Cheb-Terrab et al., 1988; Altoum et al., 2017; Mukherjee and Maiti, 1988; Altoum, 2018a). In this study we introduce solution of second order ordinary differential equations. The fundamental idea based on Toeplitz matrices with absolutely summable elements and stiffness matrix. By limiting the generality of the matrices considered, the essential ideas and results can be conveyed in a more intuitive manner without the mathematical machinery required for the most general cases. As an application the results are applied to the study of the covariance matrices and their factors of linear models of discrete time random processes (Wazwaz, 2002; Altoum, 2018c; Gupta et al., 1995; Altoum, 2018b). In this study we consider the following equation:

$$
-\frac{d}{d x}\left(k(x) \frac{d T(x)}{d x}\right)=f(x),-L<x<L
$$

$k$ : kernel conductivity of the material and $f(x)$ is the heat source for simplicity we work with $k(x)=k=$ constant. 


\section{Weak Formulation}

From Equation (1) we get:

$$
\int_{-L}^{L}-\frac{d}{d x}\left(k \frac{d T(x)}{d x}\right) v(x) d x=\int_{-L}^{L} f(x) v(x) d x
$$

where, $v$ is a test function. Let $v$ such that $v(-L)=v(L)=$ 0 ; then integrating by parts, the left hand side of Equation (2) becomes:

$$
\begin{aligned}
& \int_{-L}^{L}-\frac{d}{d x}\left(k \frac{d T(x)}{d x}\right) v(x) d x=\left[-k \frac{d T(x)}{d x} v(x)\right]_{-L}^{L} \\
& -\int_{-L}^{L}-k \frac{d T(x)}{d x} \frac{d v(x)}{d x} d x \\
& \int_{-L}^{L}-\frac{d}{d x}\left(k \frac{d T(x)}{d x}\right) v(x) d x=\int_{-L}^{L} k \frac{d T(x)}{d x} \frac{d v(x)}{d x} d x .
\end{aligned}
$$

Now the weak formulation is:

$$
\int_{-L}^{L} k \frac{d T(x)}{d x} \frac{d v(x)}{d x} d x=\int_{-L}^{L} f(x) v(x) d x
$$

we look now for $T$ and $V_{h}=\operatorname{Span}\left\{\phi_{j}\right\}$ :

$$
\phi_{j}(x)= \begin{cases}\frac{x-x_{j-1}}{\Delta x_{j}}, & x_{j-1} \leq x \leq x_{j} \\ \frac{x_{j+1}-x}{\Delta x_{j}}, & x_{j} \leq x \leq x_{j+1} \\ 0, & \text { otherwise }\end{cases}
$$

$\Delta x_{j}=h$ : uniform spacing:

$$
\phi_{j}(x)=\frac{1}{h}\left\{\begin{array}{lc}
x-x_{j-1}, & x_{j-1} \leq x \leq x_{j} \\
x_{j+1}-x, & x_{j} \leq x \leq x_{j+1} \\
0, & \text { otherwise }
\end{array}\right.
$$

and it is derivative is given by:

$$
\phi_{j}^{\prime}(x)=\frac{1}{h}\left\{\begin{array}{l}
1, \quad x_{j-1} \leq x \leq x_{j} \\
-1, \quad x_{j} \leq x \leq x_{j+1} \\
0, \quad \text { otherwise. }
\end{array}\right.
$$

Let $v=\phi_{j}(x)$ then we have:

$$
\begin{aligned}
& \int_{-L}^{L} k \frac{d T(x)}{d x} \frac{d \phi_{j}(x)}{d x} d x=\int_{-L}^{L} f(x) \phi_{j}(x) d x, \tilde{T} \simeq T \\
& \int_{x_{j-1}}^{x_{j+1}} k \frac{d \tilde{T}(x)}{d x} \frac{d \phi_{j}(x)}{d x} d x=\int_{x_{j-1}}^{x_{j+1}} f(x) \phi_{j}(x) d x
\end{aligned}
$$

where, $\tilde{T}$ is the approximate solution. Then Equation (3) becomes:

$$
\begin{aligned}
& \int_{x_{j-1}}^{x_{j}} k \frac{d \tilde{T}(x)}{d x} \frac{d \phi_{j}(x)}{d x} d x+\int_{x_{j}}^{x_{j+1}} k \frac{d \tilde{T}(x)}{d x} \frac{d \phi_{j}(x)}{d x} d x \\
& =\int_{x_{j-1}}^{x_{j+1}} f(x) \phi_{j}(x) d x \\
& \frac{1}{h} \int_{x_{j-1}}^{x_{j}} k \frac{d \tilde{T}(x)}{d x} d x-\frac{1}{h} \int_{x_{j}}^{x_{j+1}} k \frac{d \tilde{T}(x)}{d x} d x=\int_{x_{j-1}}^{x_{j+1}} f(x) \phi_{j}(x) d x .
\end{aligned}
$$

$N$ : linear elements and $N+1$ degree of freedom and therefore $N+1$ basis functions:

$$
\begin{aligned}
& \tilde{T}(x)=\sum_{j=1}^{N+1} a_{j} \phi_{j}(x) \\
& \int_{x_{j-1}}^{x_{j+1}} k \frac{d \phi_{j}}{d x} \frac{d \tilde{T}}{d x} d x=\frac{1}{h} \int_{x_{j-1}}^{x_{j}} k \frac{d \tilde{T}}{d x} d x-\frac{1}{h} \int_{x_{j}}^{x_{j+1}} k \frac{d \tilde{T}}{d x} d x
\end{aligned}
$$

using:

$$
\frac{d \tilde{T}(x)}{d x}=\sum_{j=1}^{N+1} a_{j} \frac{d \phi_{j}(x)}{d x}
$$

we have:

$$
\begin{aligned}
& \int_{x_{j-1}}^{x_{j+1}} k \frac{d \phi_{j}}{d x} \frac{d \tilde{T}}{d x} d x \\
& =\frac{k}{h}\left[\int_{x_{j-1}}^{x_{j}} \sum_{i=1}^{N+1} a_{i} \frac{d \phi_{i}(x)}{d x} d x-\int_{x_{j}}^{x_{j+1}} \sum_{i=1}^{N+1} a_{i} \frac{d \phi_{i}(x)}{d x} d x\right]
\end{aligned}
$$

we use only:

$$
\sum_{i=1}^{N+1} a_{i} \frac{d \phi_{i}(x)}{d x}=a_{j-1} \frac{d \phi_{j-1}(x)}{d x}+a_{j} \frac{d \phi_{j}(x)}{d x}
$$

and:

$$
\begin{aligned}
\sum_{k=1}^{N+1} a_{i} \frac{d \phi_{i}(x)}{d x}= & a_{j} \frac{d \phi_{j}(x)}{d x}+a_{j+1} \frac{d \phi_{j+1}(x)}{d x} \\
\int_{x_{j-1}}^{x_{j}} h \frac{d \phi_{j}}{d x} \frac{d \tilde{T}}{d x} d x= & \frac{k}{h}\left[\int_{x_{j-1}}^{x_{j}} a_{j-1} \frac{d \phi_{j-1}(x)}{d x} d x+a_{j} \frac{d \phi_{j}(x)}{d x} d x\right]- \\
& \frac{k}{h}\left[\int_{x_{j}}^{x_{j+1}} a_{j} \frac{d \phi_{j}(x)}{d x} d x+a_{j+1} \frac{d \phi_{j+1}(x)}{d x} d x\right] \\
= & \frac{k}{h}\left[\int_{x_{j-1}}^{x_{j}} \frac{\left(a_{j}-a_{j-1}\right)}{h} d x-\int_{x_{j}}^{x_{j+1}} \frac{\left(a_{j+1}-a_{j}\right)}{h} d x\right] \\
= & \frac{k}{h}\left[\frac{\left(a_{j}-a_{j-1}\right)}{h} h-\frac{\left(a_{j+1}-a_{j}\right)}{h} h\right] \\
= & \frac{k}{h}\left[2 a_{j}-a_{j-1}-a_{j+1}\right]
\end{aligned}
$$

we conclude the following: 


$$
\frac{k}{h}\left[2 a_{j}-a_{j-1}-a_{j+1}\right]=\int_{x_{j-1}}^{x_{j+1}} f(x) \phi_{j}(x) d x
$$

for given function $f$ we compute this part of our problem. Now let $j=1, \ldots, N$ our unknowns: $a_{1}, a_{2}, \ldots, a_{N+1}$.

$$
\begin{aligned}
& \tilde{T}(x)=\sum_{j=1}^{N+1} a_{j} \phi_{j}(x) \\
& \frac{k}{h}\left[\begin{array}{ccccc}
2 & 1 & 0 & 0 & 0 \\
-1 & 2 & 1 & 0 & 0 \\
\vdots & \ddots & \ddots & \vdots & \\
0 & 0 & -1 & 2 & 1
\end{array}\right]\left[\begin{array}{c}
a_{1} \\
a_{2} \\
\vdots \\
a_{N+1}
\end{array}\right]=\left[\begin{array}{c}
L\left(\phi_{1}\right) \\
L\left(\phi_{2}\right) \\
\vdots \\
L\left(\phi_{N+1}\right)
\end{array}\right]
\end{aligned}
$$

where the left matrix is Stiffness matrix and $L\left(\phi_{j}\right)=$ $\int_{x_{j-1}}^{x_{j+1}} f(x) \phi_{j}(x) d x$.

\section{Example 1}

Let $L=1, x \in[-1,1]$ and $f(x)=50 e^{x}, T(-1)=100, T(1)$ $=100$ boundary condition $\tilde{T}=100$, we will compute the value of $T$ at each nodes: $x(1)=-1, \ldots, x(N+1)=1$ :

$$
\tilde{T}=\left[\begin{array}{ccccc}
1 & 2 & \cdots & N & N+1 \\
T(1) & T(2) & \cdots & T(N) & T(N+1)
\end{array}\right]
$$

where, $\tilde{T}:$ vector $\tilde{T}(1)$ and $\tilde{T}(N+1):$ are knows, so we will determine only $\tilde{T}(2), \ldots, \tilde{T}(N)$ corresponding to the value of $\tilde{T}$ at nodes $x(2), \ldots, x(N)$, using $j=2, \ldots, N$ :

$$
\begin{aligned}
& \frac{k}{h}\left[2 a_{j}-a_{j-1}-a_{j+1}\right]=L\left(\phi_{j}\right) \\
& \frac{k}{h}\left[\begin{array}{ccccccc}
2 & 1 & 0 & 0 & 0 & 0 \cdots & 0 \\
-1 & 2 & 1 & 0 & 0 & 0 \cdots & 0 \\
\vdots & \ddots & \ddots & \ddots & \ddots & \ddots & \vdots \\
0 & 0 & -1 & 2 & 1 & 0 \cdots & 0
\end{array}\right]\left[\begin{array}{c}
a_{2} \\
a_{3} \\
\vdots \\
a_{N}
\end{array}\right]=\left[\begin{array}{c}
\frac{k}{h} a_{1}+L\left(\phi_{2}\right) \\
\vdots \\
L\left(\phi_{N-1}\right) \\
\frac{k}{h} a_{N+1} L\left(\phi_{N}\right)
\end{array}\right] \\
& \frac{k}{h} A Y=b
\end{aligned}
$$

where:

$$
b=\left[\begin{array}{c}
\frac{k}{h} a_{1} \\
0 \\
\vdots \\
0 \\
\frac{k}{h} a_{N+1}
\end{array}\right]+\left[\begin{array}{c}
L\left(\phi_{2}\right) \\
L\left(\phi_{3}\right) \\
\vdots \\
L\left(\phi_{N-1}\right) \\
L\left(\phi_{N}\right)
\end{array}\right]=\frac{k}{h}\left[\begin{array}{c}
a_{1} \\
0 \\
\vdots \\
0 \\
a_{N+1}
\end{array}\right]+\left[\begin{array}{c}
L\left(\phi_{2}\right) \\
L\left(\phi_{3}\right) \\
\vdots \\
L\left(\phi_{N-1}\right) \\
L\left(\phi_{N}\right)
\end{array}\right]
$$

In matlab, we can calculate $A$ :

$$
A=\operatorname{Toeplitz}([2,-1,0, \ldots, 0],[2,-1,0, \ldots, 0])
$$

size of $A=N-1$ because we have $a_{2}, \ldots, a_{N}$ unknowns, so how many zeros Toeplitz matrix we have $(N-1)-(2)$ $=N-3$, because we have two known coefficients 2 and 1 :

$$
A=\operatorname{Toeplitz}([2,-1, \operatorname{zeros}(1, N-3)],[2,-1, \operatorname{zero}(1, N-3)])
$$

Boundary condition:

$$
\left[\begin{array}{c}
a_{1} \\
0 \\
\vdots \\
0 \\
a_{N+1}
\end{array}\right]=\left[\begin{array}{c}
T(1,1) \\
0 \\
\vdots \\
0 \\
T(N+1,1)
\end{array}\right]
$$

nodes $=[T(1,1)$; zero $(N-3,1) ; T(N+1,1)] ; L\left(\square_{j}\right)$ must computed using integration by parts or Trapezoidal method.

First method: integration by parts:

$$
\begin{aligned}
& L\left(\phi_{j}\right)=\int_{x_{j-1}}^{x_{j+1}} f(x) \phi_{j}(x) d x \\
& =\int_{x_{j-1}}^{x_{j}} f(x) \phi_{j}(x) d x+\int_{x_{j}}^{x_{j+1}} f(x) \phi_{j}(x) d x \\
& =\int_{x_{j-1}}^{x_{j}} 50 e^{x} \frac{\left(x-x_{j-1}\right)}{\Delta x}+\int_{x_{j}}^{x_{j+1}} 50 e^{x} \frac{\left(x_{j+1}-x\right)}{\Delta x} \\
& =\frac{50}{\Delta x}\left[\int_{x_{j-1}}^{x_{j}} e^{x}\left(x-x_{j-1}\right) d x+\int_{x_{j}}^{x_{j+1}} e^{x}\left(x_{j+1}-x\right) d x\right] \\
& =\frac{50}{h}\left[e^{x_{j-1}}-2 e^{x_{j}}+e^{x_{j+1}}\right] \\
& =\frac{1}{h}\left[f x_{j-1}-2 f x_{j}+f x_{j+1}\right]
\end{aligned}
$$

Second method: We can use direct approximation of the integral without integral by parts (Trapezoidal):

$$
\begin{aligned}
& \int_{a}^{b} f(x) \simeq \frac{b-a}{2}(f(a)+f(b)) \\
& \int_{x_{j-1}}^{x_{j}} f(x) \frac{x-x_{j-1}}{\Delta x} \simeq\left[f\left(x_{j}\right) \frac{\Delta x}{\Delta x}+f\left(x_{j-1}\right) \frac{\left(x_{j-1}-x_{j-1}\right)}{\Delta x}\right] \frac{\Delta x}{2} \\
& =\left[f\left(x_{j}\right)+0\right] \frac{\Delta x}{2} \\
& =\frac{\Delta x}{2} f\left(x_{j}\right) .
\end{aligned}
$$

And: 


$$
\begin{aligned}
& \int_{x_{j}}^{x_{j+1}} f(x) \frac{\left(x_{j+1}-x\right)}{\Delta x} d x=\frac{\Delta x}{2}\left[f\left(x_{j+1}\right) \frac{\left(x_{j+1}-x_{j+1}\right)}{\Delta x}+\frac{f\left(x_{j}\right)\left(x_{j+1}-x_{j}\right)}{\Delta x}\right] \\
& =\frac{\Delta x}{2}\left[0+\frac{\Delta x}{\Delta x} f(x)\right] \\
& =\frac{\Delta x}{2} f\left(x_{j}\right) .
\end{aligned}
$$

Then:

$$
\begin{aligned}
& \int_{x_{j-1}}^{x_{j+1}} f(x) \phi_{j}(x) d x \simeq \frac{\Delta x}{2} f\left(x_{j}\right)+\frac{\Delta x}{2} f\left(x_{j}\right) \\
& =\Delta x f\left(x_{j}\right) .
\end{aligned}
$$

Conclusion second approximation of the integral:

$$
L\left(\phi_{j}\right)=\left\{\begin{array}{c}
\frac{1}{h}\left[f\left(x_{j-1}\right)-2 f\left(x_{j}\right)+f\left(x_{j+1}\right)\right], \text { integrating by parts } \\
\frac{h}{2} f\left(x_{j}\right), \text { Trapezedal rule }
\end{array}\right.
$$

Finally:

$$
T=[T(1,1), T(2,1), T(3,1), \ldots, T(N, 1), T(N+1,1)]
$$

where, the values of $N$ at $T(1,1)$ and $T(N+1,1)$ are knows the value of $N$ at $T(1,1)=-L=x_{1}$ and $T(N+1)$ $=L=x_{N+1}$ respectively. We need only calculate $[T(2,1), \ldots, T(N, 1)]$ where:

$$
T(2: N, 1)=\left(\frac{h}{k}\right) A^{-1} b .
$$

\section{Numerical Results}

To certify the proposed method, we consider the case where the data $f(x)$ is the following:

$$
f(x)=-50 e^{x}+50 x \sinh (1)+50 \cosh (1)+100
$$

Figure 1 presents the behavior the exact and numerical solution. Whereas Fig. 2 present the error between exact and numerical solution computed using finite element. The error is defined as: error $=\mid u_{\text {Analytical }}{ }^{-}$ $u_{\text {numerical }}$, for a fixed value of $N$, we get an error about $10^{-3}$. This confirm that the proposed method converge well with error about $10^{-3}$. Her, we limit ourselves to numerical convergence, Fig. 1-4 present the behavior of error for different value of $N$, its noticed that the error is sharp decreasing versus $N$.

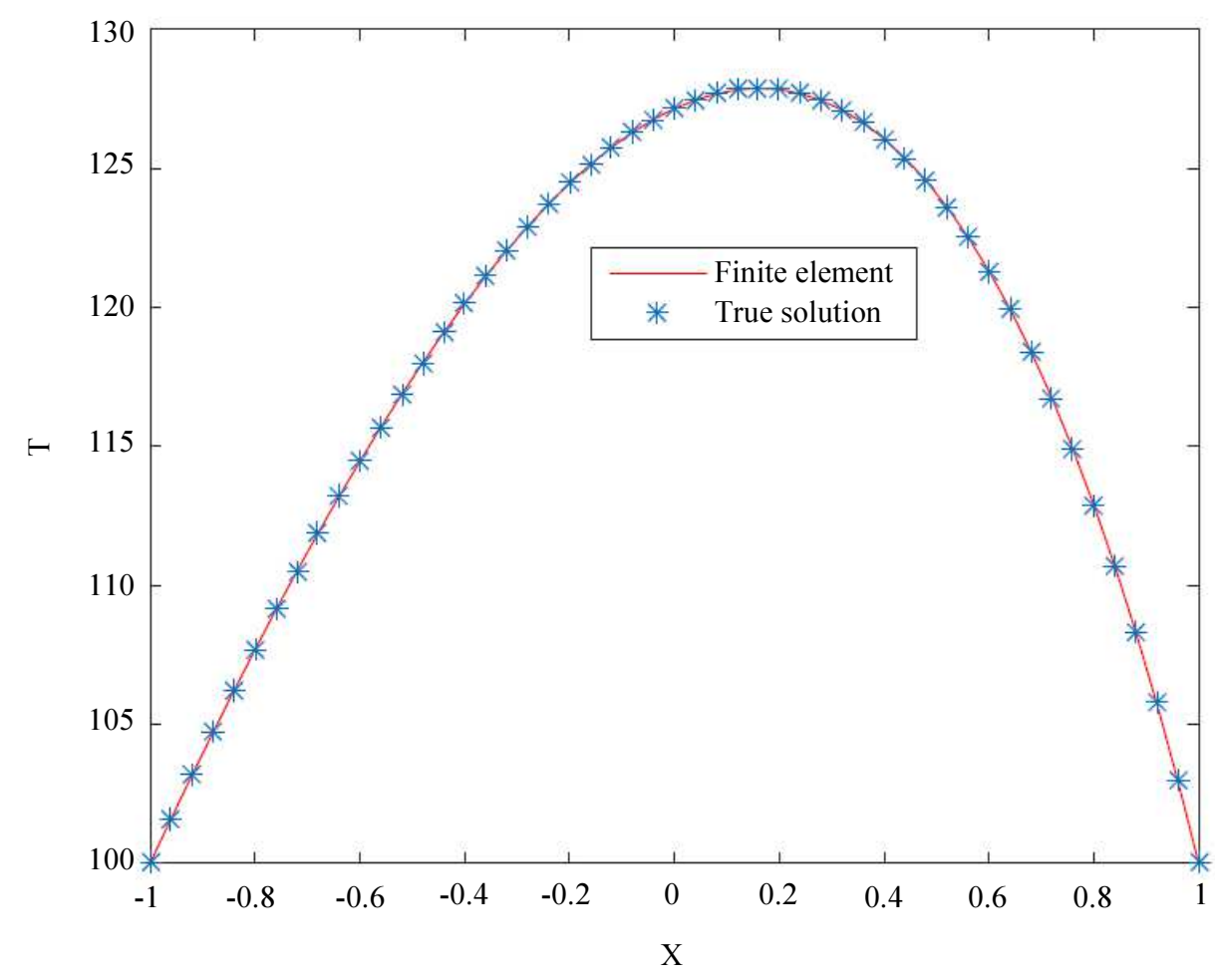

Fig. 1: Analytical solution and numerical solution using finite element 
Sami H. Altoum / American Journal of Applied Sciences 2018, 15 (8): 416.422 DOI: 10.3844/ajassp.2018.416.422

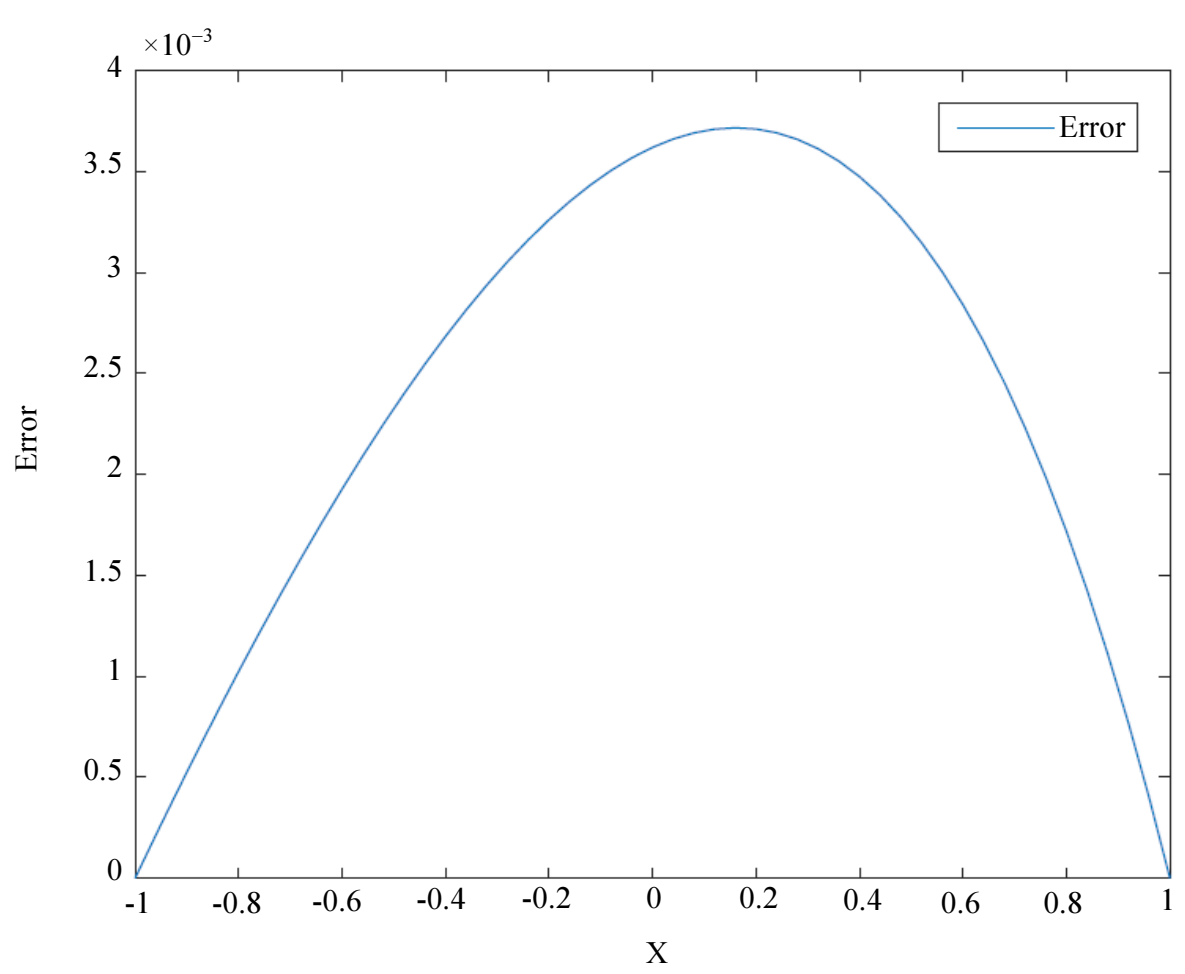

Fig. 2: Error $=\left|u_{\text {Analytical }}-u_{\text {numerical }}\right|$

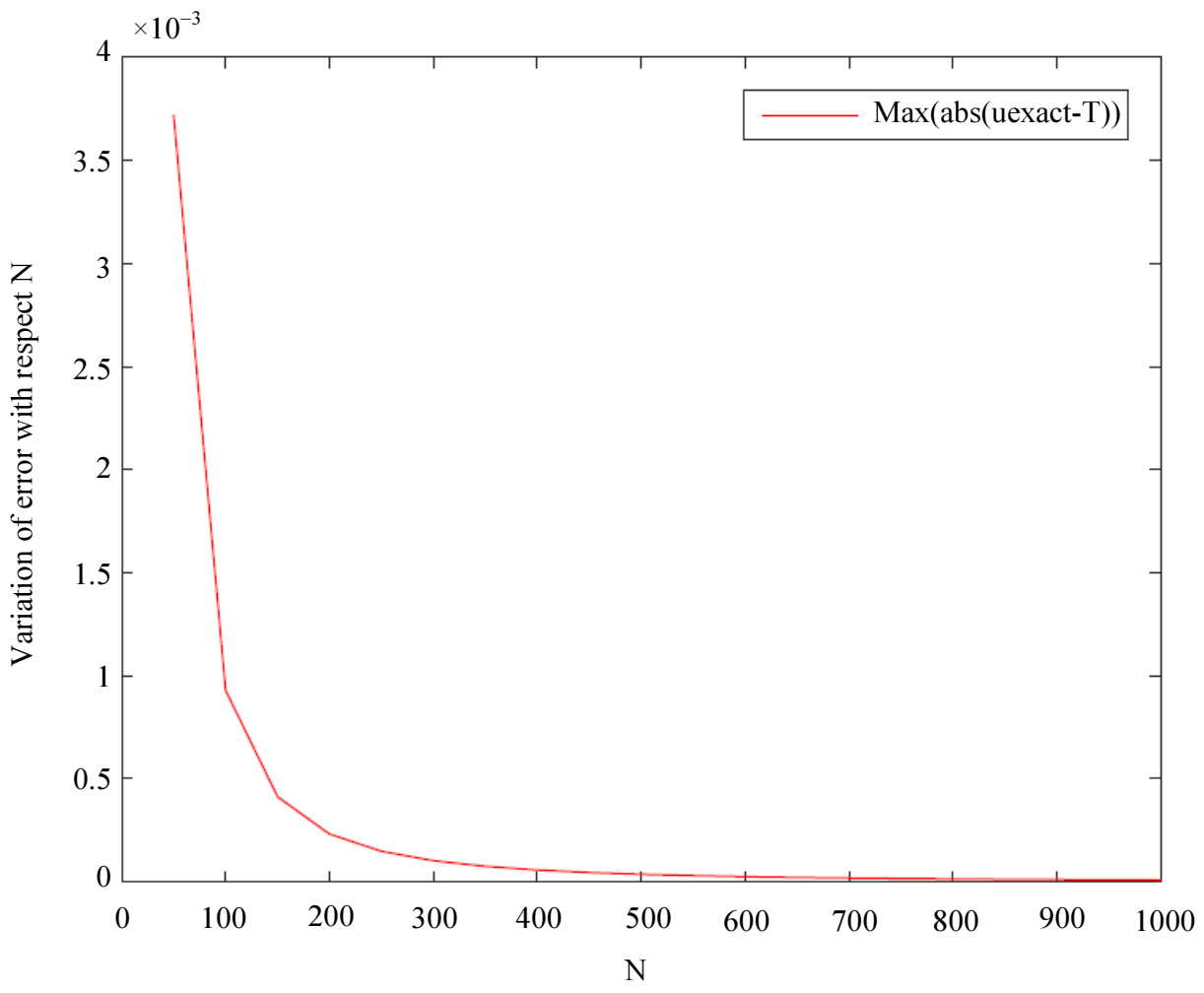

Fig. 3: Behavior of error for different value of $N$ 


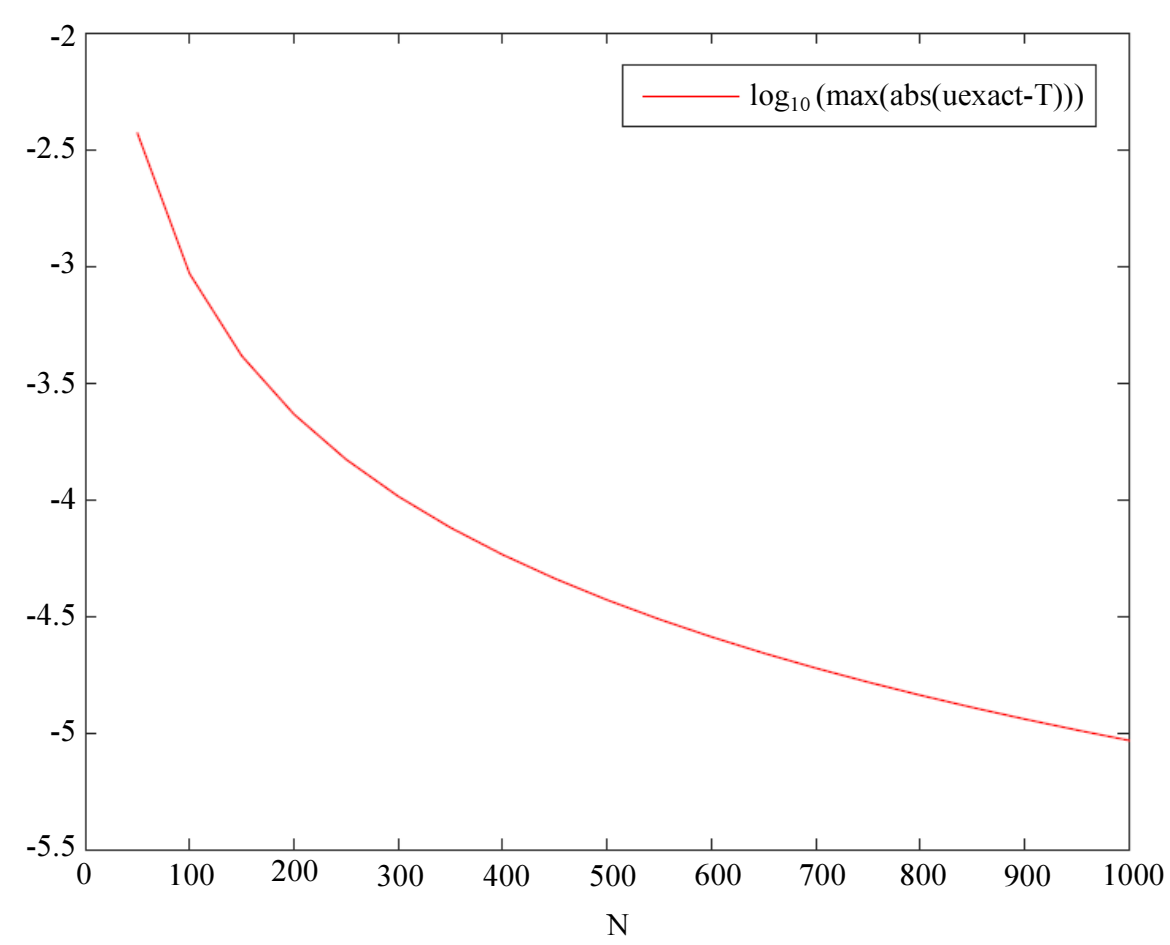

Fig. 4: $\log _{10}$ (error), for different value of $N$

\section{Discussion and Conclusion}

The strategy proposed using Stiffness and Toeplitz matrices is more exible and more faster computed to other methods, i.e., Finite element. The structure of the system obtained (Toeplitz matrix) permit to use faster matrix vector product. This method will be extended and applied for other equation, i,e. Dif- fusion problem in two dimensional case. In practice, we will obtain a very large system with Toeplitz structure. This will be solved using the main properties of the Toeplitz matrix. Finally, this paper is one of a list of papers that will be published to show the importance of Toeplitz matrices to get the system.

\section{Acknowledgement}

We would like to thanks anonymous referees for their careful reading of our manuscript and helpful suggestions.

\section{Ethics}

The author declare that there is no conict interests regarding the pub- lication of this manuscript. This article is original and contains unpublished material.

\section{References}

Agarwal, P. and A.A. El-Sayed, 2018. Non-standard finite difference and Chebyshev collocation methods for solving fractional diffusion equation. Phys. A: Stat. Mechan. Applic., 500: 40-49.

DOI: 10.1016/j.physa.2018.02.014
Altoum, S.H., 2018a. q-deformation of the square white noise Lie algebra. Trans. A. Razmadze Math. Inst., 172: 133-139.

DOI: $10.1016 /$ j.trmi.2018.01.005

Altoum, S.H., 2018b. q-sl2 and associated wave and heat equations. Am. J. Applied Sci., 15: 261-266.

Altoum, S.H., 2018c. q-Deformation of transonic gas equation. J. Math. Stat.

Altoum, S.H., H.A. Othman and H. Rguigui, 2017. Quantum white noise Gaussian kernel operators. Chaos Solitons Fractals, 104: 468-476.

DOI: 10.1016/j.chaos.2017.08.039

Cheb-Terrab, E.S., L.G.S. Duarte and L.A.C.P. da Mota, 1988. Computer algebra solving of second order ODEs using symmetry methods. Comput. Phys. Commun., 108: 90-114. DOI: 10.1016/S0010-4655(97)00132-X

Chniti, C., S.E.A. Alhazmi, S.H. Altoum, M. Toujani, 2016. DtN and NtD surface radiation conditions for two-dimensional acoustic scattering: Formal derivation and numerical validation. Applied Numerical Math., 101: 53-70. DOI: $10.1016 / \mathrm{j}$.apnum.2015.08.013

Gavin, H.P., 2014. Mathematical properties of stiffness matrices. Duke Universit.

Gupta, C.P., S.K. Ntouyas and P.C. Tsamatos, 1995. Solvability of an m-point boundary value problem for second order ordinary differential equations. J. Math. Anal. Applic., 189: 575-584.

DOI: 10.1006/jmaa.1995.1036 
Mukherjee, B.N. and S.S. Maiti, 1988. On some properties of positive definite toeplitz matrices and their possible applications. Linear Algebra Applic., 102: 211-240. DOI: 10.1016/0024-3795(88)90326-6

Ruzhansky, M., Y.J. Cho, P. Agarwal and I. Area, 2017. Advances in Real and Complex Analysis with Applications. 1st Edn., Birkhäuser,

ISBN-10: 9811043361, pp: 301.

Saoudi, K., P. Agarwal and P. Kumam, 2018. The Nehari manifold for a boundary value problem involving Riemann-Liouville fractional derivative. Adv. Diff. Equ., 2018: 263-263.

DOI: $10.1186 / \mathrm{s} 13662-018-1722-8$
Wazwaz, A.M., 2002. A new method for solving singular initial value problems in the secondorder ordinary differential equations. Applied Math. Comput., 128: 45-57.

DOI: $10.1016 / \mathrm{S} 0096-3003(01) 00021-2$

Ye, K. and L.H. Lim, 2015. Every matrix is a product of toeplitz matrices. Found. Comput. Math., 17: 577-598. 10.1007/s10208-015-9254-Z

Zhou, H. and P. Agarwal, 2017. Dix existence of almost periodic solution for neutral Nicholson blowies model. Adv. Diff. Equ., 2014: 659152-659152. DOI: $10.1155 / 2014 / 659152$ 